\title{
Cross-correlation of Circulating Sclerostin over 24 hours to PTH, Phosphate and Bone Markers in Healthy Young Men
}

\author{
${ }^{1,2}$ Santosh HS, ${ }^{1}$ Hamilton A, ${ }^{1}$ Ahluwalia R, ${ }^{3}$ Barraclough DL, ${ }^{4}$ Fraser WD, ${ }^{1}$ Vora JP,
}

${ }^{1}$ Department of Diabetes and Endocrinology, Royal Liverpool University Hospital, Prescot Street, Liverpool. L7 8XP. UK, ${ }^{2}$ Department of Endocrinology and Diabetes, Singleton Hospital, Sketty Lane, Sketty, Swansea. SA2 8QA. UK, ${ }^{3}$ Department of Clinical Chemistry, Duncan Building, Royal Liverpool University Hospital, Daulby Street, Liverpool. L69 3GA. UK, ${ }^{4}$ Department of Medicine, Norwich Medical School, University of East Anglia, Norwich. NR4 7TJ. UK

\section{Introduction}

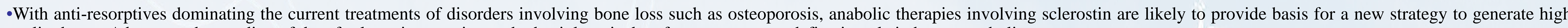
quality bone. A better understanding of the of sclerostin secretion and physiology is therefore necessary to define its role in bone metabolism.

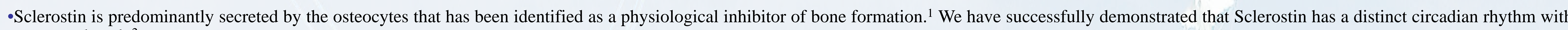
a nocturnal peak. ${ }^{2}$

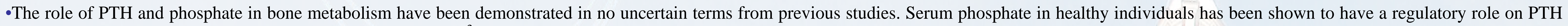
secretion, independent of its effects on calcium and vitamin D3. ${ }^{3}$

Aim

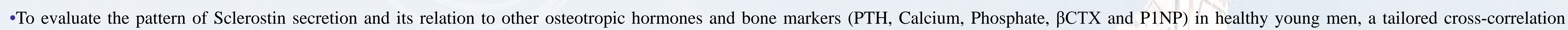
analysis was performed.

Materials and Methods

•6 healthy young men were recruited. All patients had bone densitometric evaluation using a Prodigy Oracle Fan-Beam bone densitometer (GE Medical Systems, Giles, Buckinghamshire, UK). •Sclerostin was measured by an enzyme linked immunoassay (Biomedica, Austria). All samples were assayed in duplicates. The intra and inter assay CV\% being <5\% and $12.3 \%$ respectively.

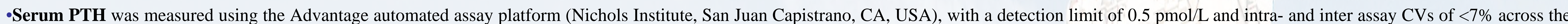
working range.

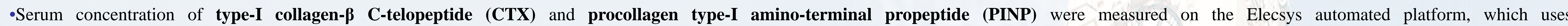

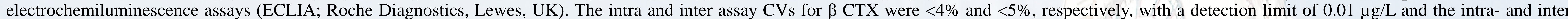
assay CVs for PINP, were $<2 \%$ and $<2.5 \%$, respectively, across the working range, with a detection limit of $4 \mu g / L$.

Statistical Analysis

-Cross-correlation analysis was performed to determine the relationships between the 24 hour profiles for Sclerostin, PTH, Calcium, Phosphate, $\beta C T X$ and P1NP.

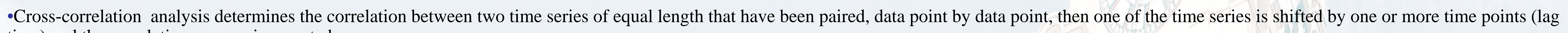
time) and the correlation process is repeated.

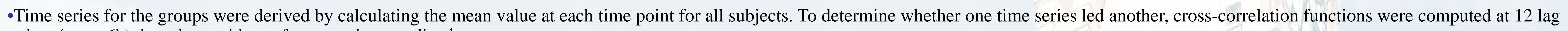
points (up to 6h), based on evidence from previous studies. ${ }^{4}$
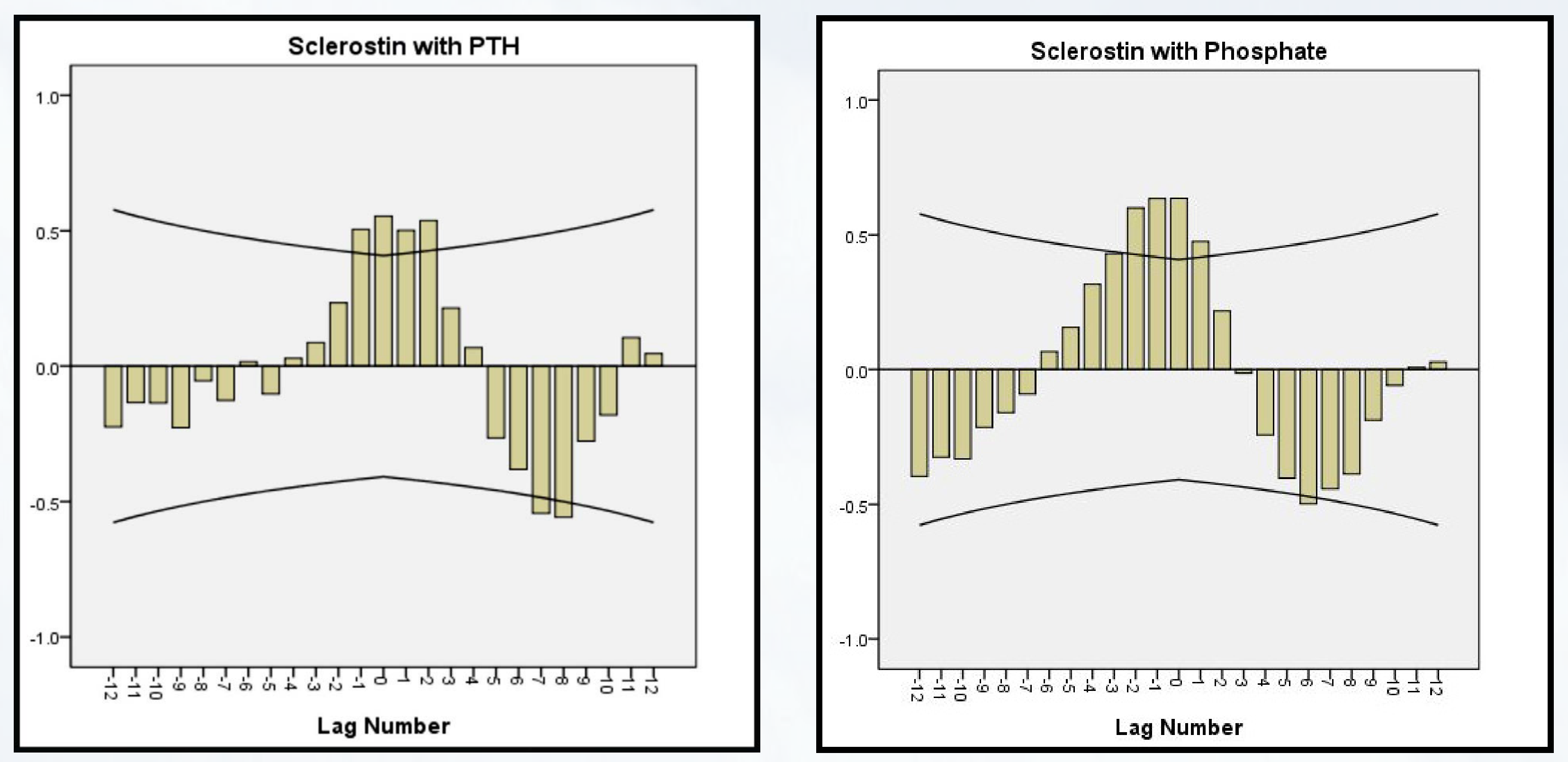

Results

-It has been demonstrated from previous studies using cross-correlation analysis in healthy individuals, that changes in serum phosphate precede the changes in PTH, which in turn precede the changes in bone resorption and formation. ${ }^{3}$

-Our results indicate that secretory patterns of sclerostin Vs. PTH and Calcium demonstrate no definite correlation during the $24 \mathrm{hr}$ period.

-A positive correlation was noted between sclerostin and phosphate, $\beta C T X, P 1 N P$ with correlation co-efficients of $0.637,0.627,0.666$ respectively. The changes in the sclerostin preceded $\beta$ CTX by $1 \mathrm{hr}$, but zero lags between sclerostin Vs phosphate and P1NP.

-The diurnal rhythms for CTx and PINP in healthy individuals demonstrated an early morning peak around $0500 \mathrm{~h}$, with a nadir at around $1400 \mathrm{~h}$, these peaks closely follow the patterns of sclerostin circadian providing further evidence to the crucial link between sclerostin and bone turnover.

\section{Summary and Conclusions}

-Despite the existence of a circadian rhythm for sclerostin it does not seem to either directly influence or be influenced by PTH secretion. It is unlikely that PTH is a modulator of sclerostin secretion.

-However, the strong correlation of sclerostin to bone markers and phosphate with a zero lag confirms that sclerostin is an important regulator of bone homeostasis

-The absence of a strong correlation between PTH secretion and sclerostin does suggest the possibility of a direct influence of yet another independent factor on sclerostin circadian rhythm and bone homeostasis.
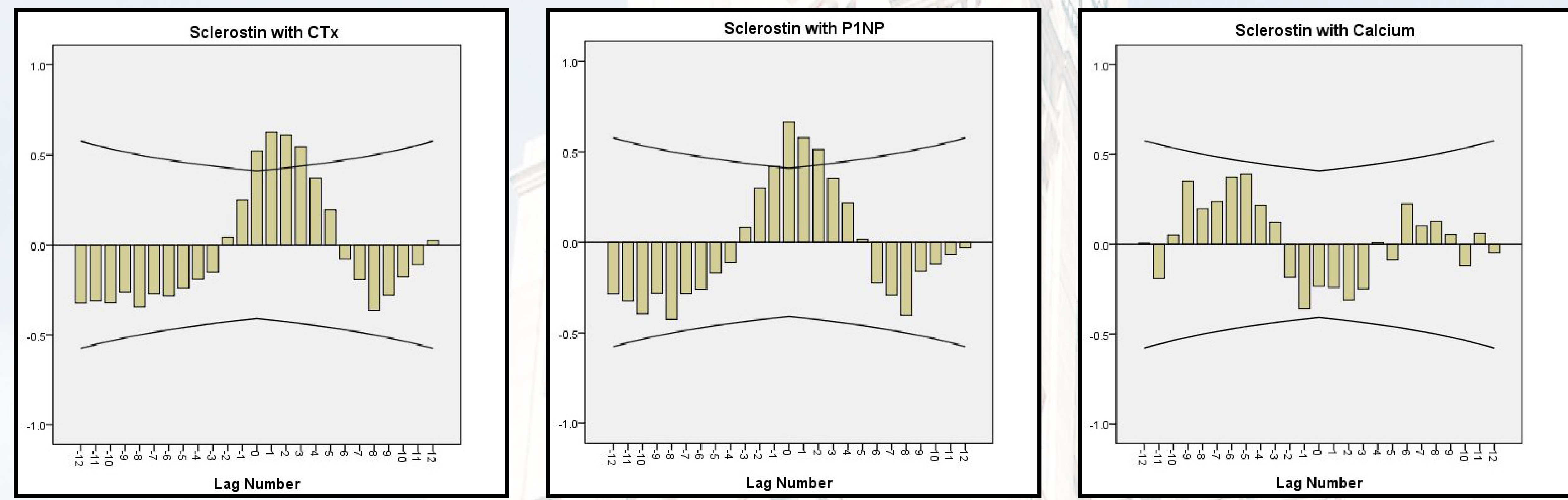

Fig. 1 Postive Correlations are demonstrated between Sclerostin and phosphate, $\beta C T X, P 1 N P$ while there was no correlation noted between Sclerostin and PTH, Calcium.
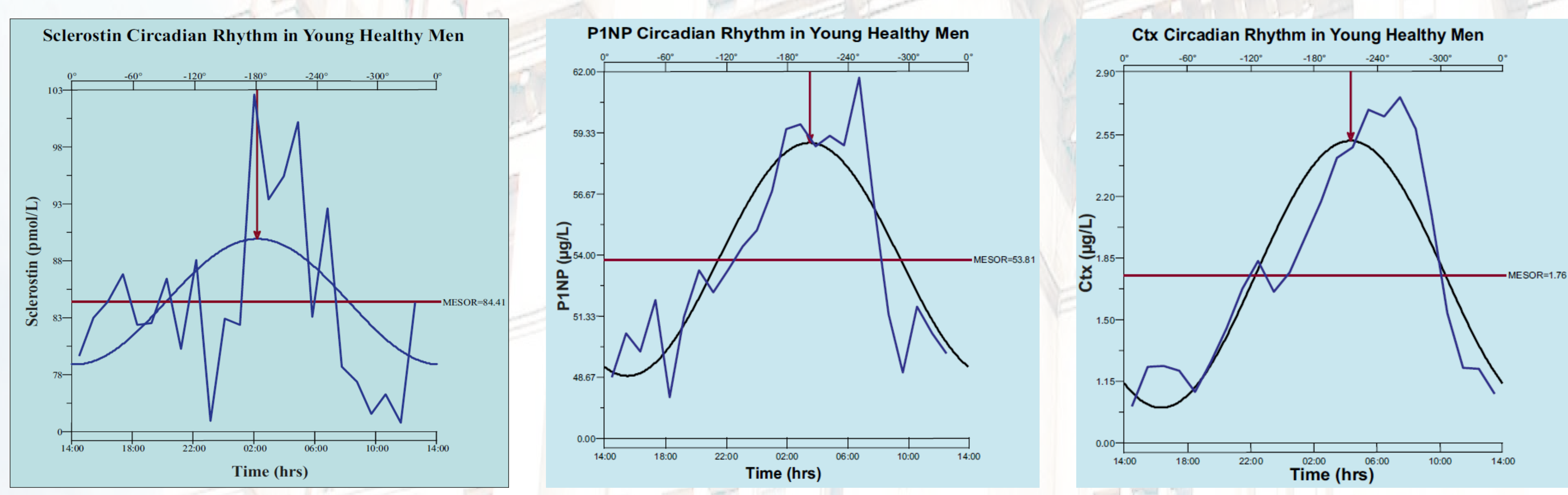

Fig. 2 An early morning peak (onset at 0100hrs) noted in the sclerostin secretion is closely followed by peak in the circulating concentrations of bone markers(P1NP and $\beta C T X)$ at 0400 and 0500 respectively. 